\title{
Premature ovarian insufficiency and fragility vertebral fracture following surgery and radiation therapy for pelvic leiomyosarcoma in a young woman.
}

\author{
Paola Villa*, Inbal Dona Amar, Clelia Cipolla, Giovanni Scambia \\ Department of Obstetrics and Gynecology, Catholic University of the Sacred Heart, Rome
}

\begin{abstract}
Although radical cancer therapies result in improvements for long-term survival, several cancer treatments that induce gonadotoxicity may put young women at risk of premature ovarian insufficiency. On the other hand, radiotherapy may result in severe impairment of bone metabolism. A 37-year-old woman underwent a surgical treatment for a retroperitoneal mesenchymal high-grade pelvic neoplasm. The retroperitoneal mass removal with preservation of the uterus and ovaries was followed by radiation therapy. After 1 year the patient showed premature ovarian insufficiency and pelvic vertebral fracture. This case emphasizes the importance of the evaluation of bone metabolism in patients who undergo cancer treatments even more if they are young and undergo possible radiation therapy.
\end{abstract}

Keywords: Radiation effects, premature ovarian insufficiency, Bone fracture.

Accepted on March 12, 2018

\section{Introduction}

The advance of long time survival in young subjects exposed to cancer therapies often brings about several later effects which must be considered for the improvements of their quality of life. Premature ovarian insufficiency (POI) is defined as an ovarian insufficiency before the age of 40 years [1]. It is characterized by termination of menstruation for at least 4 months associated with the elevation of serum follicle-stimulating hormone (FSH) concentration (FSH $>40$ IU/l) [2]. Premature ovarian insufficiency can have an influence on premature morbidity and mortality [3] that is associated with profound hypo-estrogenism, leading to a deleterious effect on different systems in the female body (1). POI can have a spontaneous or iatrogenic etiology. Spontaneous POI is referred to genetic, auto immunological, inflammatory, enzyme deficiency, metabolic, or very often idiopathic causes [4]. Iatrogenic POI takes place mainly following oncological therapy such as surgery (bilateral oophorectomy), chemotherapy and radiotherapy [4].

POI consequences can be either short or long term consequences. The short term consequences are usually due to a sudden estrogenic shortage and include vasomotor symptoms (hot flashes, night sweats and heart palpitations). The long term deleterious effects include infertility, osteoporosis, cardiovascular and neurologic disorders and an increased risk of premature death [5]. At present, while fertility-sparing treatments have been successfully attempted in selected cases of cervical, endometrial and ovarian cancer a thorough analysis of all consequences of cancer treatments should be performed in any case. Options to preserve fertility include shielding to reduce radiation damage, fertility preservation when undergoing cytotoxic treatments, cryopreservation of ovarian tissue, assisted reproduction techniques and fertility-sparing surgical procedures [6,7].

In the female population, several treatments may deplete the ovarian pool and the ovarian endocrine function inducing a POI
$[6,8]$. The risk of POI following chemotherapy and surgery has been well studied while radiation therapy long term effects must be better clarified and considered.

\section{Case Report}

A 37 year-old woman came to our Centre in November 2012 for an amenorrhea started in January 2012. Her history began three years before when she underwent a medical consult for the presence of a swelling in the right inguinal region, present for about a year and gradually increasing in absence of other symptoms. The following abdominal echography revealed a solid mass of about $18 \mathrm{~cm}$ in the pelvis. Computed Tomography (CT) scans showed a bulky solid mass with heterogeneous density that caused compression on the uterus which appeared dislocated on the other side without apparent infiltration. No appreciable regional lymphadenopathies had been noted. The Magnetic Resonance Imaging (MRI) showed a pelvic mass causing caudal and lateral bladder compression and displacement of the uterus and ovaries.

Patient was initially subjected to surgery to remove the pelvic mass which was retroperitoneal and did not involve the uterus or ovaries. The mass removal required the resection of part of the sigmoid colon and the resection and reconstruction of a segment of the left external iliac artery and vein. The histologic exam provided a diagnosis of mesenchymal high-gradeneoplasm, leiomyosarcoma. The stage of disease was pT2bN0Mx because the vessels, the connective adipose tissue and lymph nodes were free of metastases. Subsequently the patient was treated with three cycles of radiotherapy on the pelvic tumor field (total dose of $5040 \mathrm{cGy}, 180 \mathrm{cGy} /$ day) with a specific dose of $5940 \mathrm{cGy}$ on a field following the external iliac course up to the aortic bifurcation (the irradiation field included the left margin of the lumbar spine from T11 to L4).

After surgery she had 3 regular menstrual cycles then there was a period of amenorrhea for about 1 year. The patient approached 
Citation: Villa P, Amar ID, Cipolla C, et al. Premature ovarian insufficiency and fragility vertebral fracture following surgery and radiation therapy for pelvic leiomyosarcoma in a young woman. Gynecol Reproduct Endocrinol -UK. 2018;2(1):11-14

our center in order to clarify the origin of the persistent amenorrhea. Among the symptoms, the patient mentioned amenorrhea, some mild menopausal symptoms, increased nocturnal sweating and a slight recent lumbar pain.

The clinical and the endocrine status were tested. A bone mineral density measurement for lumbar spine (L2-L4 area) with dual energy X-ray absorptiometry (DXA) was performed and contemporary Morphometry-DXA exam of the spine aimed at clarifying the lumbar pain.

The hormonal analysis showed an increase of FSH (68.5 mUI/ $\mathrm{ml})$ as well as the very low E2 $(14 \mathrm{pg} / \mathrm{ml})$ and AMH levels $(<0.4 \mathrm{ng} / \mathrm{ml})$ confirming the diagnosis of early menopause due to POI. Vitamin D levels were in the normal range for our laboratory as well as all other bone marker parameters (PTH $31.9 \mathrm{pg} / \mathrm{ml}$; Osteocalcin $44.9 \mathrm{ng} / \mathrm{ml}$, phosphor $3.2 \mathrm{mg} / \mathrm{ml}, \mathrm{Ca}$ $10.1 \mathrm{mg} / \mathrm{ml}$, Vit D $13.7 \mathrm{ng} / \mathrm{ml}$ ). Pelvic US examination showed a normal uterus and small ovaries without follicular growth. The patient did not have any previous period of amenorrhea nor was treatment with glucocorticoid and her BMI 26.5 at the time of the visit. The DXA evaluation showed a low bone mineral density with a BMD of $0.922 \mathrm{~g} / \mathrm{cm}^{2}$ which was equivalent to a $\mathrm{T}$ score of $-1,1$ and to a $Z$ score of $-1,3$ therefore, our patient was diagnosed with osteopenia.

The DXA-Morfometry (Figure 1) showed 18\% vertebral wedge deformation at $\mathrm{T} 11^{\text {th }}$ vertebral body and $20 \%$ deformation of the $\mathrm{L} 4^{\text {th }}$ with a mild vertebral crush indicating vertebral fractures despite the presence of osteopenia.

\section{Discussion and Conclusion}

We report a unique case of insufficiency vertebral fractures following premature menopause due to post-operative radiation therapy for a pelvic leiomyosarcoma, appearing only 14 months after surgical extensive pelvic intervention without ovarian involvement.

The association of surgery and radiotherapy in young survivors of pelvic or gynecological cancer may, not surprisingly, induce gonadotoxicity leading to infertility and in some cases to premature menopause $[8,9]$. In this case while the ovaries were not involved in the surgical procedure they may have undergone severe damage following the irradiation therapy and in particular the left ovary may have had a further injury resulting from the altered vascularization due to surgery.

Several findings indicated that the risk of POI increased significantly with increasing doses of abdominal pelvic irradiation. In particular, the risk of nonsurgical premature menopause was elevated among survivors exposed to the highest doses of ovarian irradiation (>1000 cGy) $[9,10]$. A recent mathematical model employed by Wallace et al suggested that the dose required to destroy $50 \%$ of the immature oocytes (LD50) is less than 2 Gy $[11,12]$. It is important to note that with novel radiation techniques, including IMRT (Intensity modulated radiation therapy) and proton radiotherapy, the ovaries may be spared from significant radiation, thus mitigating the potential adverse effects on fertility $[13,14]$. However, there are still few published clinical or dosimetric data that directly address this issue.
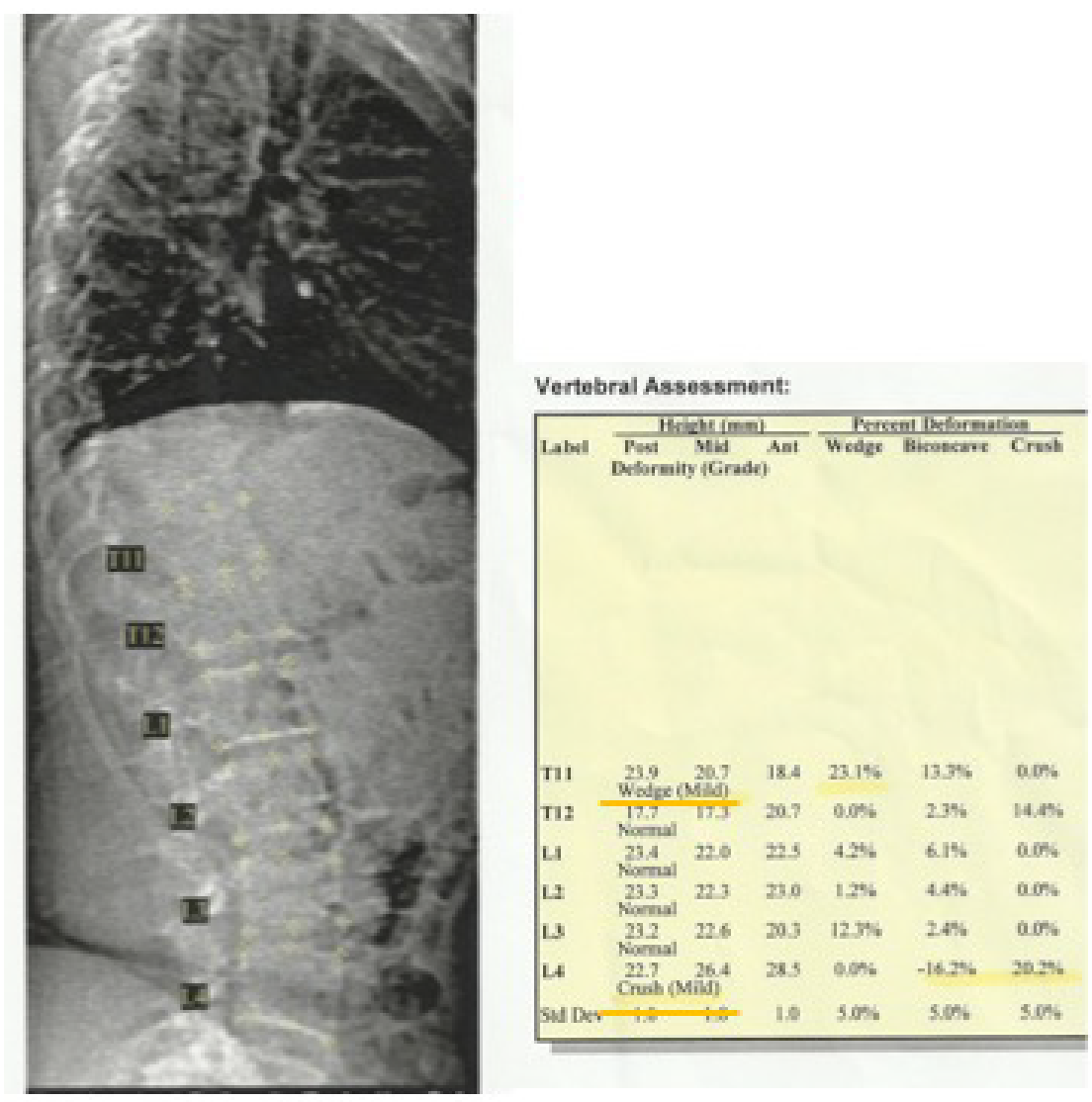

Figure 1: DXA morphometry assessmemnt: T11 vertebral mild grade wedge deformation and L4 crush with $20 \%$ deformation. 
Survivors who lose ovarian function during cancer therapy or shortly after its completion are classified as having acute ovarian failure and patients younger than 40 who experience menopause are classified as having premature menopause [15]. These patients show the acute hormonal impairment of menopause with all the consequences of acute estrogen function loss including osteoporosis $[16,17]$.

In our case the contemporary combined surgery and radiotherapy treatment can account for the development of premature menopause. The rapid decrease in estrogen levels accelerated bone loss and may have had significant effects on bone metabolism. In particular in the early years after menopause, BMD quickly decreases because of the early mobilization of calcium from the skeleton particularly from the spine. This acute bone disorder predisposes patients to insufficiency fractures (IF), a type of stress fractures that occur after physiological stress.

External-beam pelvic irradiation directly damages bones and may lead to fractures independent from the levels of bone mineral density $[10,18]$. At first, few studies focused on the incidence of radiation induced pelvic insufficiency fractures $[11,19]$. Many of these studies conducted after therapy for gynecologic malignancies or for colon-rectum cancer in postmenopausal women indicated an incidence rate of about 30-40\% [20-23].

Recently the potential morbidity of pelvic radiation has been better clarified. Different threshold-doses for IF due to external-beam doses have been estimated in about 45-50 Gy $[11,13,19,24]$ while the cumulative 5 -year risk of IF for postoperative pelvic radiotherapy (RT) in gynecological cancer ranged from $13 \%$ to $5.1 \%$ [16,25-27]. Radiation even has an indirect effect associated with vascular changes [20,22]. Therefore one of the main factors responsible for the effect on irradiated bone is injury of the microvasculature of mature bone, where microcirculation occlusion results in an increased susceptibility to stress fracture [10,18].

Ultimately associated risk factors for osteoporosis such as age, gender, body size, dietary calcium, regular exercise, drug, caffeine and alcohol consumption, Vit-D insufficiency and other metabolic disturbances are closely correlated with the development of IF and may contribute to lower BMD which could expose patients to risk of fractures.

This 37 year old woman was not considered a candidate for a BMD test before RT; in fact she had no risk factors for osteoporosis and had not been previously treated with chemotherapy and/or corticosteroid therapy. After the diagnosis of premature menopause the bone mineral density measurement for the lumbar spine (L2-L4 area) with DXA was performed and showed a low BMD of $0.922 \mathrm{~g} / \mathrm{cm}^{2}$ in relationship to an equally aged population. Therefore the DXA-morphometry allowed us to clarify the recent pain in the lumbar spine and shows a thoracic and lumbar vertebral deformation demonstrating severe osteoporosis.

In conclusion, RT tolerance for vertebral bone tissue must be carefully evaluated in young cancer patients at risk of premature menopause. The BMD test and the evaluation of clinical osteoporosis risk before cancer treatments may contribute to the prevention of insufficiency fractures. If low BMD is demonstrated, the contemporaneous DXA-morphometric exam may help discover vertebral fracture or deformity.

\section{Consent}

Written informed consent was obtained from the patient for the publication of this case report and any accompanying images.

\section{References}

1. Luisi S, Orlandini C, Regini C, et al. Premature ovarian insufficiency: From pathogenesis to clinical management. J Endocrinol Invest 2015;38:597-603.

2. Vujovic S, Brincat $\mathrm{M}$, Erel $\mathrm{T}$, et al. EMAS position statement: Managing women with premature ovarian failure. European menopause and andropause society. Maturitas 2010;67:91-3.

3. Shuster LT, Rhodes DJ, Gostout BS, et al. Premature menopause or early menopause: Long-term health consequences. Maturitas 2010;65:161-166.

4. Nelson LM, Clinical practice. Primary ovarian insufficiency. N Engl J Med 2009;360:606-614.

5. Løkkegaard E, Jovanovic Z, Heitmann BL, et al. The association between early menopause and risk of ischaemic heart disease: Influence of hormone therapy. Pedersen AT Maturitas 2006;53:226-233.

6. Salama M, Winkler K, Murach KF, et al. Female fertility loss and preservation: Threats and opportunities. Ann Oncol 2013;24:598-608.

7. Jemal A, Clegg LX, Ward E, et al. Annual report to the nation on the status of cancer, 1975-2001, with a special feature regarding survival. Cancer 2004;101:3-27.

8. Gracia CR, Sammel MD, Freeman E, et al. Impact of cancer therapies on ovarian reserve. Fertil Steril 2012;97:134-140.

9. Martinez A, Poilblanc M, Ferron G. et al. Fertilitypreserving surgical procedures, techniques. Best Pract Res Clin Obstet Gynaecol 2012;26:407-424.

10. Chiarelli AM, Marrett LD, Darlington G, et al. Early menopause and infertility in females after treatment for childhood cancer diagnosed in 1964-1988 in Ontario, Canada. Am J Epidemiol 1999;150:245-254.

11. Wallace WH, Thomson AB, Kelsey TW, et al. The radio sensitivity of the human oocyte. Hum Reprod 2003;18:117121.

12. Sklar CA, Mertens AC, Mitby $P$, et al. Premature menopause in survivors of childhood cancer: A report from the childhood cancer survivor study. J Natl Cancer Inst 2006;98:890-896.

13. Small W Jr, Mell LK, Anderson P, et al. Consensus guidelines for delineation of clinical target volume for intensity-modulated pelvic radiotherapy in postoperative treatment of endometrial and cervical cancer. Int J Radiat Oncol Biol Phys 2008;71:428-434. 
Citation: Villa P, Amar ID, Cipolla C, et al. Premature ovarian insufficiency and fragility vertebral fracture following surgery and radiation therapy for pelvic leiomyosarcoma in a young woman. Gynecol Reproduct Endocrinol -UK. 2018;2(1):11-14

14. Lee SJ, Schover LR, Partridge AH, et al. American society of clinical oncology recommendations on fertility preservation in cancer patients. J Clin Oncol 2006;24:29172931.

15. Meirow D, Nugent D. The effects of radiotherapy and chemotherapy on female reproduction. Hum Reprod Update 2001;7:535-543.

16. Vega EM, Egea MA, Mautalen CA. Influence of the menopausal age on the severity of osteoporosis in women with vertebral fractures. Maturitas 1994;19:117-124.

17. Adriaens I, Smitz J, Jacquet P, et al. The current knowledge on radio sensitivity of ovarian follicle development stages. Hum Reprod Update 2009;15:359-377.

18. Hitoshi IKO, Shunsuke F. Pelvic bone complications following radiation therapy of gynecologic malignancies: Clinical evaluation of radiation-induced pelvic insufficiency fractures Gynecol Oncol 2006;103:1100-1104.

19. Blomlie V, Rofstad EK, Talle K, et al. Incidence of radiation-induced insufficiency fractures of the female pelvis: Evaluation with MR imaging. AJR 1996;167;12051210.

20. Tokumaru S, Toita T, Oguchi $M$, et al. Insufficiency fractures after pelvic radiation therapy for uterine cervical cancer: An analysis of subjects in a prospective multiinstitutional trial, and cooperative study of the Japan Radiation Oncology Group (JAROG) and Japanese
Radiation Oncology Study Group (JROSG). Int J Radiat Oncol Biol Phys 2012;84:e195-e200.

21. Baxter N, Habermann EB, Tepper JE, et al. Risk of pelvic fractures in older women following pelvic irradiation. JAMA 2005;20:2587-2593.

22. Wo JY, Viswanathan AN. Impact of radiotherapy on fertility, pregnancy, and neonatal outcomes in female cancer patients. Int J Radiat Oncol Biol Phys 2009;73:1304-1312.

23. Wallace WH, Thomson AB, Saran F, et al. Predicting age of ovarian failure after radiation to a field that includes the ovaries. Int J Radiat Oncol Biol Phys 2005;62:738-744.

24. Fu AL, Greven KM, Maruyama AY, et al. Radiation osteitis and insufficiency fractures after pelvic irradiation for gynecologic malignancies. Am J Clin Oncol 1994;17:248-254.

25. Oh D, Huh SJ, Nam H, et al. Pelvic insufficiency fracture after pelvic radiotherapy for cervical cancer: Analysis of risk factors. Int J Radiat Oncol Biol Phys 2008;70:1183-1188.

26. Shih KK, Folkert MR, Kollmeier MA, et al. Pelvic insufficiency fractures in patients with cervical and endometrial cancer treated with post-operative pelvic radiation. Gynecol Oncol 2013;128:540-543.

27. Green DM, Sklar CA, Boice JD Jr, et al. Ovarian failure and reproductive outcomes after childhood cancer treatment: Results from the childhood cancer survivor study. J Clin Oncol 2009; 27:2374-2381.

\section{*Correspondence to:}

Paola Villa

Department of Obstetrics and Gynecology

Catholic University of the Sacred Heart

Rome

Italy

Tel: 0635404269

E-mail: paola.villa@policlinicogemelli.it 